\title{
Changes in Public Healthcare Entitlement and Healthcare Utilisation among the Older Population in Ireland
}

Anne Nolan ${ }^{12}$, Yuanyuan Ma ${ }^{13}$, Patrick Moore ${ }^{1}$

1. The Irish Longitudinal Study on Ageing

2. Economic and Social Research Institute (ESRI), Dublin, Ireland

3. Institute for the Study of Labor (IZA), Bonn, Germany

On behalf of the TILDA team

October 2016 
Copyright (C) The Irish Longitudinal Study on Ageing 2016

The Irish Longitudinal Study on Ageing

Lincoln Place

Trinity College Dublin

Dublin 2

Tel: +35318964120

Email: tilda@tcd.ie

Website: www.tilda.ie

https://www.doi.org/10.38018/TildaRe.2016-03 


\section{Key Findings}

- The current Irish system of financing and public healthcare entitlements is unusual in a European context, with the requirement for a large proportion of the population to pay the full cost of general practitioner (GP) care at the point of use a particular concern.

- Using nationally-representative data on the over 50s from the Irish Longitudinal Study on Ageing (TILDA), we find that changes in public healthcare entitlements are associated with changes in the utilisation of GP services.

- Gaining a full medical or GP visit card is associated with 1.3 extra GP visits per annum, an increase of approximately 43 per cent.

- For those who lose a full medical or GP visit card, the number of GP visits falls by 1.2 visits per annum, which is equivalent to a fall of approximately 29 per cent.

- There is limited evidence of effects on other measures of healthcare utilisation, i.e., changing public healthcare user fees, most notably for GP care, does not result in a significant change in the utilisation of other types of healthcare such as preventive or hospital services.

- However, removing user fees for public healthcare results in a significant increase in the number of medications dispensed.

- From a current policy perspective, with free GP care now extended automatically to all under 6 s and over 70 s, and further plans to extend free GP care to all under $18 \mathrm{~s}$, the key question is whether the increase in GP utilisation we observe upon receipt of a full medical/GP visit card reflects an increase in beneficial care.

- Further analysis is required to unpick the complex causal relationships between costsharing, utilisation and health outcomes in order to inform future policy proposals in the Irish context. 


\section{Acknowledgements}

We would like to acknowledge the vision and commitment of our funders, Irish Life, the Atlantic Philanthropies and the Department of Health, which is providing funding on behalf of the state. We would also like to state that any views expressed in this report are not necessarily those of the Department of Health or of the Minister of Health. We would also like to thank the TILDA participants without whom this research would not be possible. 


\section{Contents}

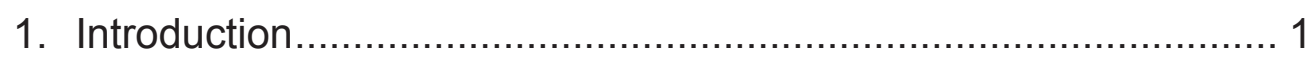

2. Institutional Background ..................................................... 3

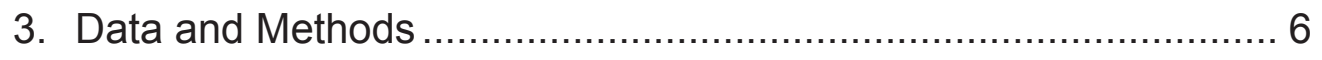

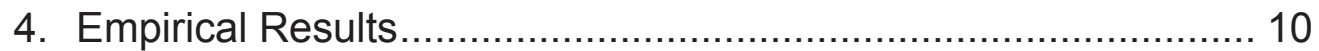

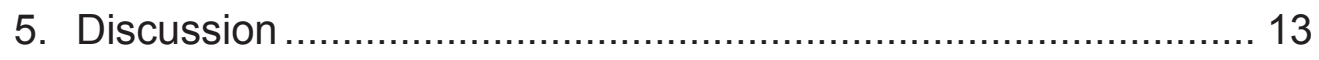

6. References ................................................................ 16 


\section{Introduction}

Equity of access to healthcare is regarded as a key objective of national and international health policy. Most countries subscribe to the principal that healthcare should be financed in accordance with ability to pay, and delivered on the basis of need. Indeed, current Irish health policy includes a commitment to 'access to quality preventative, primary, curative, rehabilitative and end-of-life healthcare that is timely and affordable for everyone' $[1 ; 63]$.

The current Irish system of healthcare financing relies predominately on public sources of finance, with smaller contributions from private health insurance (PHI) and direct out-of-pocket payments by individuals. This system of financing, and the associated structure of public healthcare entitlements, has been criticised on equity and efficiency grounds. While the proportion of overall out-of-pocket contributions to total healthcare financing is comparable with the OECD average [2], for certain services they are much more significant than is the case elsewhere. In particular, the use of direct out-of-pocket payments to finance general practitioner (GP) care by the majority of the population is unusual in a European context [3]. In addition, the role of private health insurance (PHI) in facilitating faster access to public hospital services for those with $\mathrm{PHI}$ over those without $\mathrm{PHI}$ but with equivalent health needs has also been highlighted $[4,5]$.

In summer 2015, free GP care for all children aged under 6 years of age, and all adults aged 70+ years was introduced. In May 2016, the new Government announced a commitment to an extension of free GP care to all those under 18 years of age [1]. In the context of extensions in free GP care, it is crucial to understand current patterns of healthcare utilisation, not only for highlighting the extent to which the current system leads to financial barriers to accessing healthcare services, but also for forecasting the likely demand implications of reform proposals so that policymakers can cost the proposals and plan effectively.

Providing care free at the point of use has an inherent tension between the benefit of reducing an individual's exposure to financial risk, and the disadvantage of potentially increasing an individual's use of low-value or ineffective health care [6]. However, identifying the effect of insurance on healthcare utilisation is complicated by the fact that 
individuals who have greater need for healthcare are more likely to purchase/be in receipt of insurance [7]. Simply comparing the utilisation levels of those with and without insurance is misleading: any difference in utilisation could be because of insurance, or it could occur because individuals in different insurance states may have very different health risks and other characteristics. Even with the inclusion of appropriate control variables in a statistical model, there is always the risk of unmeasured differences between groups biasing any comparisons [8]. However, evidence from a variety of settings, including from a number of randomised control trials, demonstrates that insurance is associated with increased healthcare utilisation [6, 8-14].

In this report, we focus on the older population. While still a relatively young population by EU standards [15], the population of Ireland is ageing. By 2046, approximately 21 per cent of the Irish population will be aged 65 years or older, and approximately 7 per cent will be aged 80 years or older (the corresponding figures for 2011 were 11.6 per cent and 2.8 per cent respectively) [16]. Increasing concerns over the sustainability of expenditure on health care, social care and pensions, and the resulting need for difficult decisions around the allocation of resources, requires an appropriate evidence base with which to evaluate policy proposals. Data from The Irish Longitudinal Study on Ageing (TILDA), a nationally-representative study of the over 50 s in Ireland, is used in this report to examine the determinants of healthcare utilisation among the older population. Longitudinal data such as that available in TILDA, which follows the same individuals through time, allow us to examine the effect of changing public healthcare entitlements on healthcare utilisation. This allows us to generate accurate estimates of the likely demand impacts of changes to public healthcare entitlements in this population.

The report is structured as follows. Section 2 provides further details on the current system of public healthcare entitlements in Ireland. Section 3 describes the TILDA data in greater detail and outlines the statistical methods used in this report. Section 4 presents empirical results while Section 5 discusses the findings, drawing out implications for policy. 


\section{Institutional Background}

Currently, there are two main categories of entitlements to public health services in Ireland, as illustrated in Table 1. Those in Category I (full medical cardholders) are entitled to free public health services (including inpatient and outpatient hospital care, GP care and other primary and community care services), but must pay a co-payment of $€ 2.50$ per prescription item, up to a maximum of $€ 25$ per family per month. Those in Category II are entitled to subsidised public hospital services, but must pay the full cost of GP services and other primary and community care services, and prescription medicines up to a deductible of $€ 144$ per family per month. In 2010, the average cost of a GP consultation was estimated at $€ 51$ [17]. In October 2005, the GP visit card was introduced; GP visit cardholders have the same entitlements to free GP care as Category I individuals, but the same entitlements to all other public health services (including prescription medicines) as Category II individuals. GPs in Ireland act as gatekeepers for secondary care, and the same GPs treat both Category I and II patients. 


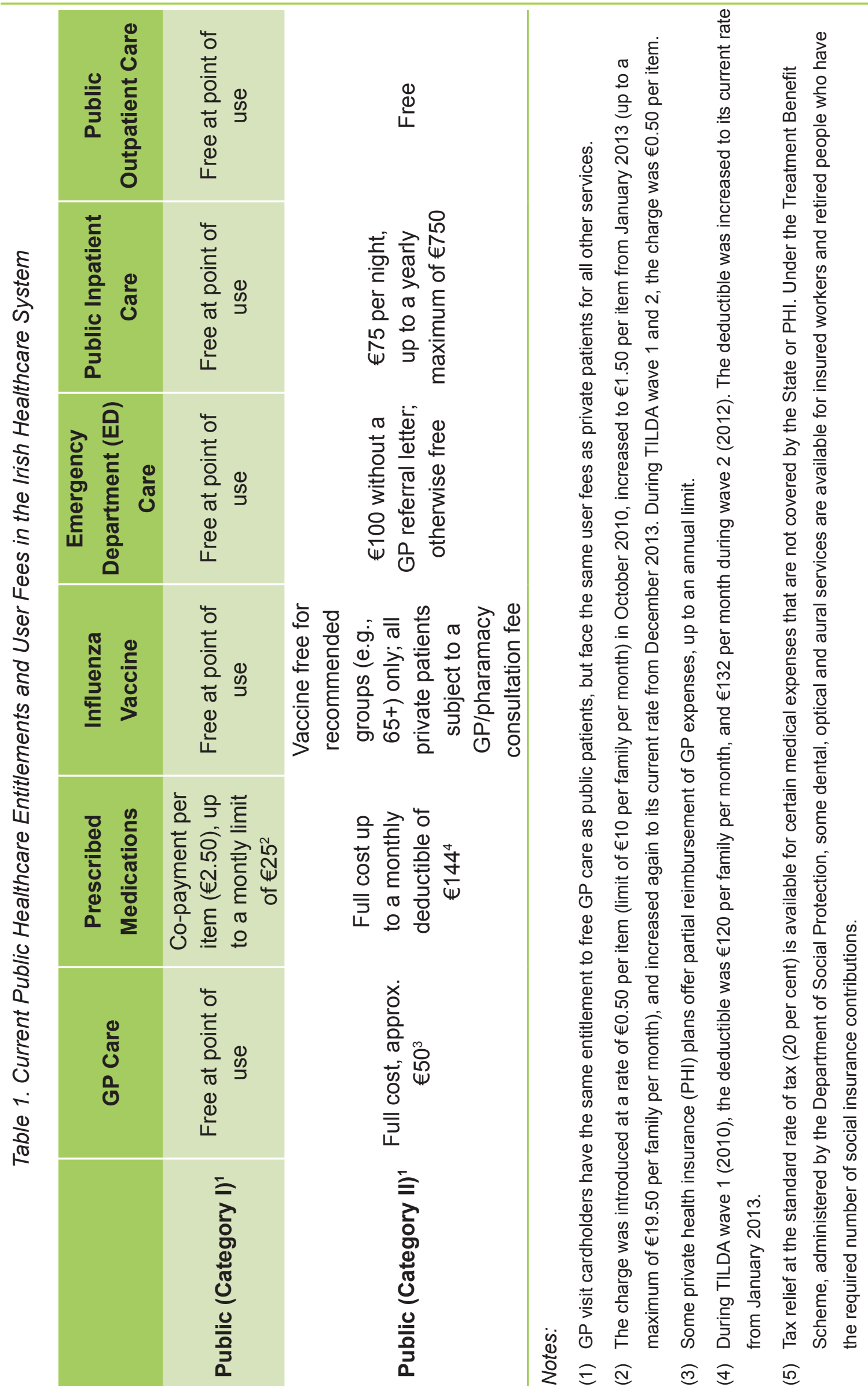


Eligibility for a full medical/GP visit card is assessed primarily on the basis of an income means test. The income thresholds for the GP visit card are 50 per cent higher than for the full medical card. In certain cases, individuals who are otherwise ineligible for a full medical/GP visit card may be granted a card on a 'discretionary' basis, if they have particular health needs which would cause them undue hardship [18]. In November 2015, 5.6 per cent of full medical cards, and 9.6 per cent of GP visit cards were issued on a 'discretionary' basis [19]. As noted in Section 1, children under 6 years of age, and adults aged $70+$ years, are now entitled automatically to a GP visit card, regardless of income. As of November 2015 (i.e., after the extension to all under 6s and over 70s), 37.4 per cent of the population had a full medical card and 9.2 per cent had a GP visit card [19].

A further layer of complexity is added to the Irish system by the existence of PHI. Currently approximately 46 per cent of the population have $\mathrm{PHI}$ [20], which mainly provides cover for private or semi-private acute hospital services (which may be delivered in public hospitals), but which increasingly offers partial reimbursement of certain primary care expenses (e.g., GP visits, routine dental care, physiotherapy, etc.). Full medical card and GP visit cardholders may take out $\mathrm{PHI}$ if they wish, and while the numbers doing so are generally small, a higher proportion of the older population have both a full medical/GP visit card and PHI (termed 'dual' cover). In 2010, 36 per cent of the over 50s had a full medical or GP visit card (without PHI), 16 per cent had 'dual cover' (i.e., a full medical or GP visit card and $\mathrm{PHI}$ ), 37 per cent had $\mathrm{PHI}$ only and 11 per cent had 'no cover' [21]. 


\section{Data and Methods}

\subsection{The Irish Longitudinal Study on Ageing (TILDA)}

To investigate the impact of the particular system of Irish healthcare financing and entitlements on healthcare utilisation among the older population, we use data from the first two waves of TILDA. TILDA is a nationally representative sample of communitydwelling individuals aged 50 years and over in Ireland (and their spouses or partners of any age) (i.e., individuals living in nursing homes or other institutions were excluded at baseline). The study is modelled closely on other international longitudinal studies of ageing, such as the US Health and Retirement Study, the Survey of Health, Ageing and Retirement in Europe and the English Longitudinal Study on Ageing. Data collection for the first wave took place over the period October 2009 to February 2011, when 8,504 individuals were sampled, of which 8,175 were aged $50+$ years. Further information on the sample design is available in [22-24].

The dataset contains a rich set of variables on the health and socio-economic circumstances of older people. Data were collected via computer-aided personal interviewing (CAPI), and the majority of participants also completed a self-completion questionnaire (SCQ) which was designed to collect more sensitive information on issues such as relationships, expectations and mood/feelings. At Wave 1, approximately 73 per cent of all participants also underwent a nurse-led health assessment (either in a dedicated health assessment centre or at home). The second wave of TILDA (without the health assessment) was carried out between April 2012 and January 2013, when 87.5 per cent of participants in Wave 1 were successfully followed up [21]. In total, 6,949 individuals aged $50+$ with complete information on public healthcare entitlements participated in both waves of the study and form the sample used in this report.

\subsection{Methods}

Previous work by the TILDA team using wave 1 data has demonstrated the association between public healthcare entitlements and the utilisation of GP services using multivariate regression methods $[25,26]$. In this report, we extend these analyses to analyse the 
impact of a change in public healthcare entitlements on changes in healthcare utilisation. We do this using a difference-in-difference propensity score matching (PSM) approach. Difference-in-difference PSM is a technique that analyses the impact of a 'treatment' (e.g., the change in public healthcare entitlement) on an outcome of interest (e.g., the change in the number of GP visits). 'Treatment' and 'control' individuals are matched on observable characteristics (e.g. marital status, education, employment status, household income, health, etc.) measured at wave 1, and their outcomes compared. This allows us to control for baseline differences in characteristics between those who change their public healthcare entitlement status between wave 1 and 2 of TILDA, and those who do not, while at the same time controlling for possible unobserved differences in characteristics between the two groups (e.g., attitudes towards risk, genetic endowments, etc.). The method ensures that individuals who are identical at wave 1, but who differ only in their exposure to changing public healthcare entitlement status are compared, thus generating accurate estimates of the impact of changes in public healthcare entitlements on healthcare utilisation. This method is described in greater detail in [27].

\subsection{Public Healthcare Entitlements}

Our key variable of interest is the change in public healthcare entitlement between wave 1 and 2 of TILDA. Previous research on the Irish system of public healthcare entitlements among the older population has identified four mutually exclusive groups [21]:

- Full medical or GP visit cardholders, without PHI

- Full medical or GP visit cardholders, with PHI ('dual cover') $\}$ Category I (public)

- PHI only

- No medical card, GP visit card or PHI ('no cover')

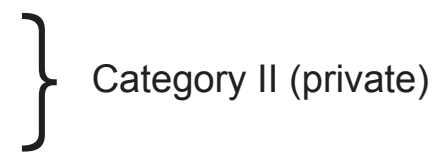

As the proportion of TILDA participants with a GP visit card is so small (just 1.8 per cent), we aggregate full medical and GP visit cardholders in our sample. Ideally, we would like to examine all possible transitions (e.g., moving from 'no cover' in wave 1 to 'full medical/GP visit card' in wave 2), but we are constrained by the small number of observations for some transitions (e.g., just 29 individuals moved from having PHI only in wave 1 to a full medical 
or GP visit card without $\mathrm{PHI}$ in wave 2). We therefore examine two key transitions: public to private ('change 1'), and private to public ('change 2') (Table 2). Change 1 examines the impact of gaining a full medical or GP visit card (i.e., removing user fees for public healthcare services, principally GP care) on healthcare utilisation, and is directly relevant to current Irish government policy proposals, while change 2 examines the impact of losing a full medical or GP visit card (i.e., introducing user fees for public healthcare services, principally GP care) on healthcare utilisation.

Table 2. Transitions in Public Healthcare Entitlements in over 50s between 2010 and 2012

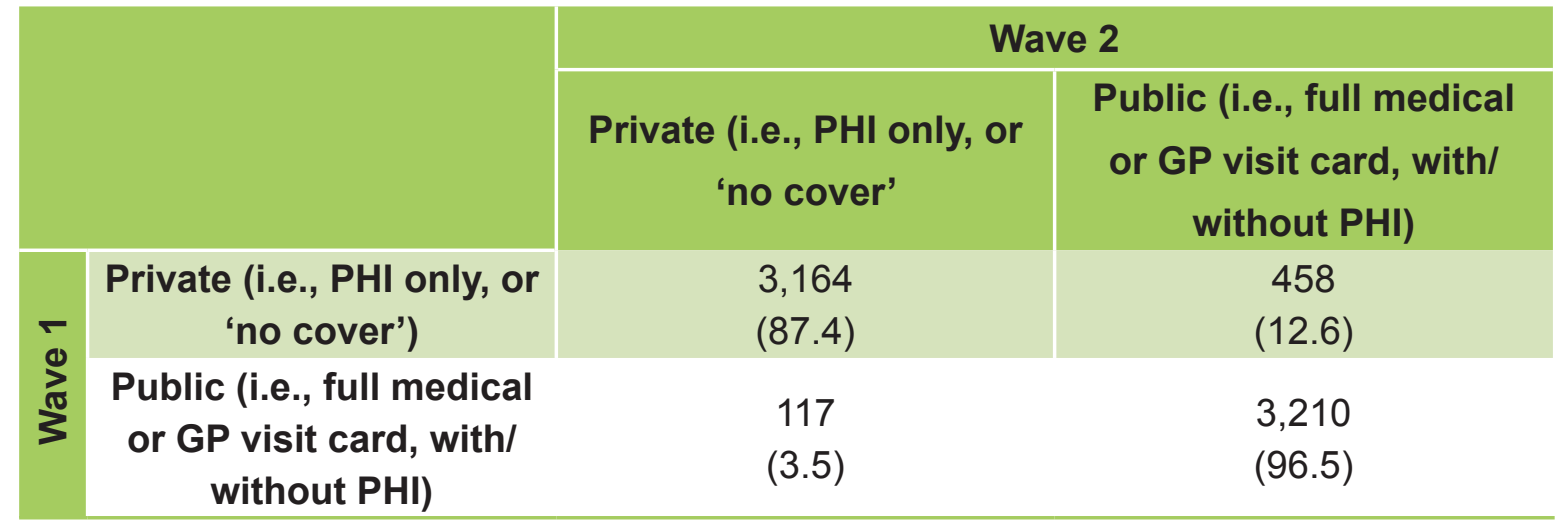

Notes:

(1) Based on the balanced sample, i.e., those present in both wave 1 and wave 2 of TILDA.

(2) The data in the table are interpreted as follows: of those who were classified as private patients (i.e., PHI only or 'no cover') in wave 1, 3,164 or 87.4 per cent were also classified as private patients in wave 2, while 458 or 12.6 per cent had transitioned to being classified as a public patient (i.e., having a full medical or GP visit card) by wave 2 .

\subsection{Outcome Variables}

TILDA contains detailed information on numerous types of healthcare utilisation. In this report, we examine the impact of changing public healthcare entitlements on the following types of healthcare:

- GP visits

- Medications

- Influenza vaccination

- Emergency department (ED) visits

- Outpatient visits

- Inpatient nights in hospital 
Table 3 presents averages for each of the six measures of healthcare utilisation examined in this report.

Table 3. Healthcare Utilisation Summary Statistics

\begin{tabular}{|l|c|c|}
\hline & Wave 1 & Wave 2 \\
\hline GP visits, mean/year & 3.9 & 3.9 \\
\hline Number of medications, mean/year & 2.6 & 3.3 \\
\hline Influenza vaccination (\%) & 49.3 & 56.8 \\
\hline ED visits, mean/year & 0.25 & 0.24 \\
\hline Outpatient visits, mean/year & 1.40 & 1.56 \\
\hline Inpatient nights, mean/year & 1.05 & 1.39 \\
\hline
\end{tabular}

Notes:

(1) Based on the balanced sample, i.e., those present in both wave 1 and wave 2 of TILDA.

(2) Population weights are employed.

(3) For GP visits, ED visits, outpatient visits and inpatient visits, the top 1 per cent of outlying observations are excluded.

As we are examining changes in utilisation between wave 1 and 2 of TILDA, all outcome variables are expressed in the form of the difference between wave 1 and 2. For GP visits, ED visits, outpatient visits and inpatient hospital nights, the relevant measure refers to the number of visits/nights in the last twelve months. For the measure of preventive healthcare utilisation (flu vaccine), the measure refers to 'ever had' the vaccine. For medications, the number of medications (which also includes supplements) currently being taken is recorded. While TILDA contains information on the utilisation of various State-funded primary and community services (such as physiotherapy, occupational therapy, etc.), the absence of information on the use of these services in the private sector means that a full picture of utilisation across the public and private sectors cannot be ascertained. In addition, in wave 1, no information is provided on the frequency of State-funded primary and community service use in the past twelve months.

All information on healthcare utilisation is self-reported by the participant. The potential for measurement error when healthcare utilisation information is recorded in this way is wellrecognised in the literature $[28,29]$, but the limited evidence on the agreement between self-reported data and administrative records is mixed [30-32]. While we acknowledge this potential limitation of the outcome variables, as we are using the difference in utilisation between wave 1 and 2 as the outcome variables, the length of the recall period should be less of an issue for this analysis, as the over- or under- reporting should be a fixed individual characteristic that should not vary over time. 


\section{Empirical Results}

\subsection{Descriptive Patterns}

Before presenting the results of the statistical analyses, it is useful to set out the current distribution of public healthcare entitlements across the older population and how they have changed between wave 1 and wave 2 of TILDA.

As noted in Section 2.1, in 2010 (TILDA wave 1), 36 per cent of the over 50s had a full medical or GP visit card (without PHI), 16 per cent had 'dual cover' (i.e., a full medical or GP visit card and PHI), 37 per cent had PHI only and 11 per cent had 'no cover'. By 2012 (TILDA wave 2), the proportions of the older population with a full medical or GP visit card (without PHI) and 'no cover' had changed only marginally (to 39 per cent and 10 per cent respectively), although there was a significant increase in the proportion of the older population with dual cover' (to 18 per cent), and a significant reduction in the proportion with PHI only (to 33 per cent) [21] (see also Figure 1).

Figure 1. Public Healthcare Entitlements, 50+ Population, 2010-2012

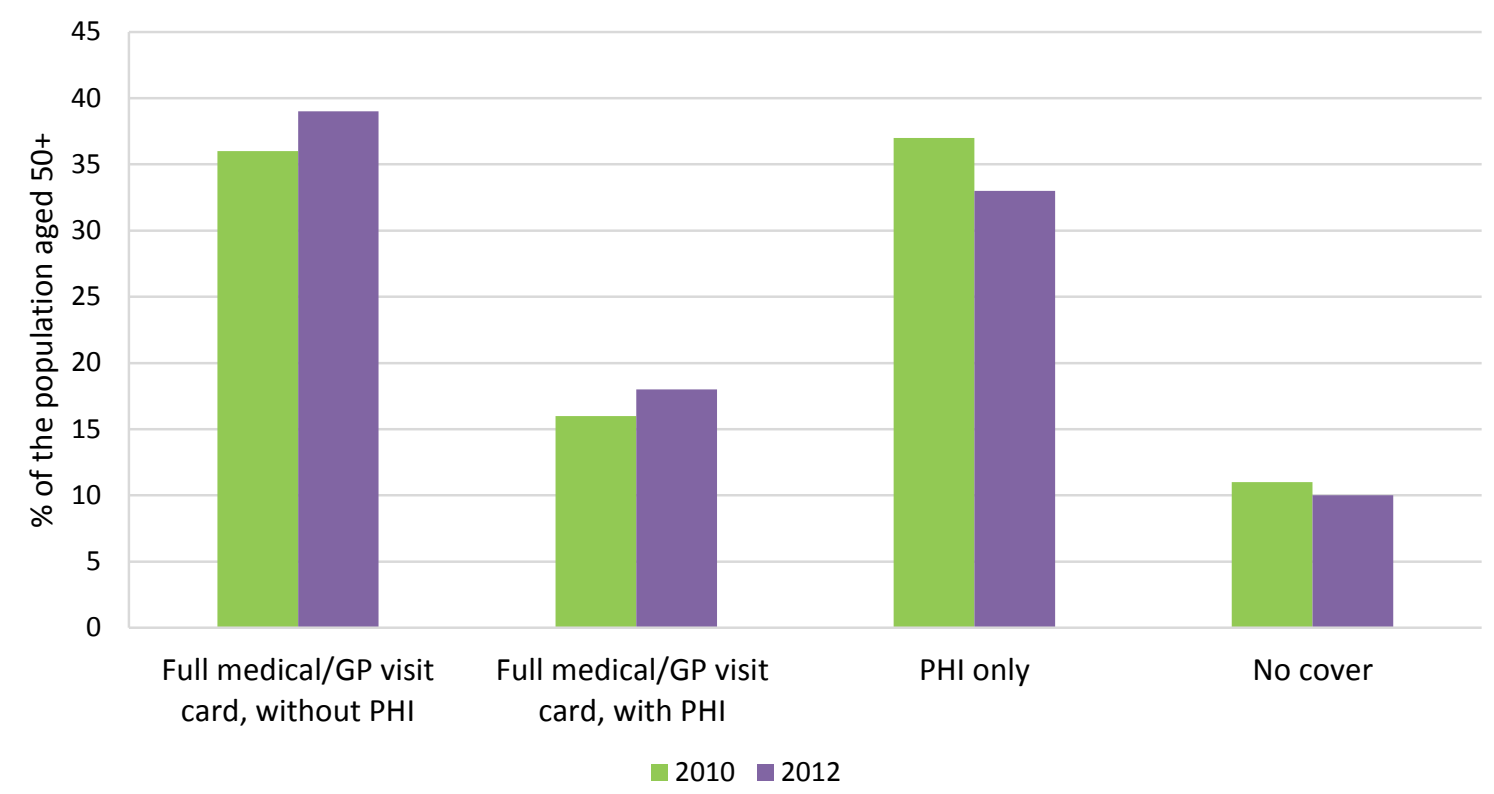

Notes:

(1) Full medical/GP visit cardholders with PHI are also referred to as having 'dual cover'.

(2) The first two groups are public or Category I patients, while the last two groups (PHI only and 'no cover') are private or Category II patients. 


\subsection{Modelling Results}

Table 4 presents the results for change 1, i.e., gaining a full medical or GP visit card, and change 2, i.e., losing a full medical or GP visit card. Results are presented for each of the six indicators of healthcare utilisation.

For change 1, individuals who gained a full medical or GP visit card are compared with similar individuals who remained as private patients between wave 1 and 2 of TILDA, while for change 2, individuals who lost a full medical or GP visit card are compared with similar individuals who remained as public patients between wave 1 and 2 of TILDA. Those in change group 1 therefore benefitted from the removal of user fees for many public health services, principally GP care and prescription medicines, while those in change group 2 became liable for user fees for many public healthcare services, principally GP services and prescription medicines (see Table 1 for further details).

The results indicate that gaining a full medical or GP visit card is associated with approximately 1.3 extra GP visits per annum. Compared to the wave 1 level of GP visiting for this group (3.03), this equates to an approximate increase of 43 per cent in the annual number of GP visits for those who gain a medical or GP visit card. Looking at change 2, we find that losing a full medical or GP visit card is associated with approximately 1.2 fewer GP visits per annum. Compared to the wave 1 level of GP visiting for this group (4.1), this equates to an approximate decrease of 29 per cent in the annual number of GP visits for those who lose a medical or GP visit card.

The results for the other indicators of healthcare utilisation indicate no difference in the probability of having had a flu vaccination, or in the number of ED visits, outpatient visits or inpatient nights in hospital for either change. However, gaining a full medical or GP visit card is associated with an increase in the number of medications that are regularly taken (i.e., approximately 0.4 extra medications, or an approximate increase of 16 per cent). 
Table 4. Difference-in-Difference Propensity Score Matching Estimates of Changes in Healthcare Utilisation

\begin{tabular}{l|c|c} 
& $\begin{array}{c}\text { Change 1: } \\
\text { Gaining a full medical/ } \\
\text { GP visit card }\end{array}$ & $\begin{array}{c}\text { Change 2: } \\
\text { Losing a full medical/ } \\
\text { GP visit card }\end{array}$ \\
\hline GP visits & 1.30 & -1.21 \\
\hline Medications & $(0.28)^{* * *}$ & $(0.54)^{* *}$ \\
\hline Flu vaccination & 0.37 & -0.29 \\
& $(0.11)^{* * *}$ & $(0.17)^{*}$ \\
\hline ED visits & 0.01 & 0.02 \\
& $(0.02)$ & $(0.04)$ \\
\hline Outpatient visits & 0.00 & -0.04 \\
& $(0.06)$ & $(0.40)$ \\
\hline Inpatient hospital nights & 0.46 & -1.15 \\
& $(0.33)$ & $(0.96)$ \\
\hline
\end{tabular}

Notes:

(1) The results indicate the change in the outcome measure (GP visits, number of medications, etc.) associated with a change in entitlement status. For example, those gaining a full medical or GP visit card had 1.3 extra GP visits per annum compared with those who remained as private patients between wave 1 and wave 2 .

(2) All specifications are estimated on common support.

(3) Kernel matching reports bootstrap standard errors (50 replications) in parentheses. ${ }^{* * *} p<0.01,{ }^{* *} p<0.05$, * $p<0.1$ 


\section{Discussion}

Equity of access to healthcare is regarded as a key objective of national and international health policy. The current Irish system of financing and public healthcare entitlements is unusual in a European context, with the requirement for a large proportion of the population to pay the full cost of GP care at the point of use a particular concern [3-5]. Given this system, and current reform proposals, it is crucial to investigate the effect of the current system on healthcare utilisation patterns, and in particular, the demand implications of changing public healthcare entitlements.

Using nationally-representative data on the over 50s from the Irish Longitudinal Study on Ageing (TILDA), our results indicate that changes in public healthcare entitlements are associated with significant changes in the utilisation of GP services. Gaining a full medical or GP visit card is associated with 1.3 extra GP visits per annum, an increase of approximately 43 per cent over the baseline level of GP visiting. For those who lose a full medical or GP visit card, the number of GP visits per annum falls by approximately 1.2, which is equivalent to a fall of approximately 29 per cent. Importantly, these effects control for baseline differences in characteristics between the various groups (i.e., those who gain a full medical or GP visit card are compared only with those who are similar to them in terms of baseline characteristics such as age, sex, income, health, etc. but who do not change their public healthcare entitlement status between waves). Of direct relevance to current Irish policy proposals, these results suggest that the removal of user fees for public healthcare services, principally in the form of GP user fees, is associated with an increase in the use of GP services.

It is also crucial to analyse the potential offsetting effects of insurance/cost-sharing on other sectors (i.e., does the introduction of user fees for GP care lead to an increase in ED visits?). Our results suggest that such effects are minimal, in that changes in public healthcare entitlements, while associated with significant changes in GP utilisation, are not associated with any significant changes in the probability of a flu vaccination, or the number of ED visits, outpatient visits or inpatient nights. However, gaining a full medical or GP visit card is associated with a significant increase in the number of medications regularly taken. 
It is important to point out a number of caveats in interpreting these results. First, due to sample size, not all possible transitions in public healthcare entitlements could be examined. For example, for policy purposes, it would be useful to examine the impact of moving from 'no cover' to a GP visit card. However, in order to ensure sufficient sample size, we had to aggregate categories of entitlement to examine broad categories, i.e., public and private, and the associated transitions. This means that for example, the effect of gaining a full medical or GP visit card on GP visiting is likely to be greater than the impact of gaining a GP visit card only due to the lack of prescription drug coverage with a GP visit card, and evidence from other settings that prescription drug coverage affects GP visiting rates [33-35]. As further waves of TILDA data become available, we will be able to extend our analysis to consider more transitions.

Second, our statistical approach cannot account for changes in certain characteristics between waves that might also (partly) explain the change in healthcare utilisation. For example, an individual could gain a full medical or GP visit card because they became ill and could no longer work; the increase in GP visiting observed could therefore partly be due to their poorer health status and greater health needs rather than solely the incentive effects of gaining a full medical or GP visit card. Therefore, the estimated effects are likely to be upper bounds on the true effects of gaining/losing a full medical or GP visit card.

Third, with two waves of data spanning a two year period, we cannot evaluate whether the observed effects are likely to be sustained over a longer follow-up period. It may be the case that those who become eligible for a full medical or GP visit card exhibit 'pent-up' demand whereby the initial demand response to the removal of user fees is not sustained in the longer term [14, 36]. Further waves of data are required to investigate this possibility.

Finally, to fully evaluate the impact of insurance/cost-sharing on total health expenditure, an assessment of the impact of insurance/cost-sharing on health is also necessary. However, two waves of data are not sufficient to facilitate an analysis of the effect of changes in public healthcare entitlements on health. Internationally, the literature on the broader question of whether insurance/cost-sharing impacts on health is ambiguous [37], partly due to the short length of the follow-up period available in many studies. In addition, our results examine only the impact of changing public healthcare entitlements on utilisation, and cannot say anything about potential supply-side responses (e.g., how GPs or other providers may respond to a change in demand), or whether current capacity on the supply side would be sufficient to meet the extra healthcare utilisation associated with extensions in insurance. 
The estimates for change 1, i.e., gaining a full medical or GP visit card, are of direct relevance to current Irish health policy. All individuals over the age of 70 years are now entitled to a GP visit card, regardless of their income. From a policy perspective, the key question is whether the increase in GP utilisation we observed upon receipt of a full medical/GP visit card reflects an increase in beneficial care. However, distinguishing between 'effective' and 'ineffective' care is very difficult, and requires much more detailed data on diagnoses, length of consultation, etc. [38]. Despite the move towards the removal of user fees for GP care in current Irish health policy, user fees for other services (e.g., prescription drugs) have been introduced (and subsequently increased) in recent years. Patient cost-sharing has been a key method of healthcare expenditure control in many countries because it assumes that people will value what they pay for, and as a result will reduce their use of ineffective healthcare when they are required to pay for it. However, healthcare is not a usual consumer good, and the presence of uncertainty and information asymmetry means that there is now considerable evidence demonstrating that user fees reduce both effective as well as ineffective healthcare use, particularly in terms of prescription drug use $[8,10,37,39,40]$. However, that is not to say that patient costsharing should never be used in healthcare settings; there is increasing attention now on value-based pricing, i.e., adjusting user fees to reflect the value of care rather than applying user fees to broad categories of care (e.g., eliminating cost-sharing for childhood immunisations) $[3,6,41]$. Further analysis is required to unpick the complex causal relationships between cost-sharing, utilisation and health outcomes in order to inform future policy proposals in the Irish context. 


\section{References}

1. Government of Ireland, A Programme for Parternship Government. 2016, Stationery Office: Dublin.

2. OECD, Health at a Glance 2015: OECD Indicators. 2015, OECD: Paris.

3. Evetovits, T., et al., Health system responses to financial pressures in Ireland: policy options in an international context. 2012, European Observatory on Health Systems and Policies: Brussels.

4. Ruane, F., Report of the Expert Group on Resource Allocation and Financing in the Health Sector. 2010, Department of Health and Children: Dublin.

5. Smith, S., The Irish health basket: a basket case? The European Journal of Health Economics, 2010. 11(3): p. 343-350.

6. Swartz, K., Cost-sharing: effects on spending and outcomes, in Research Synthesis Report No.20. 2010, The Robert Wood Johnson Foundation: Princeton.

7. Buchmueller, T., et al., Access to Physician Services: Does Supplemental Insurance Matter? Evidence from France. Health Economics, 2004. 13(7): p. 669-687.

8. Baicker, K. and D. Goldman, Patient Cost-Sharing and Healthcare Spending Growth. Journal of Economic Perspectives, 2011. 25(2): p. 47-68.

9. Keeler, E., Effect of Cost Sharing on Use of Medical Services and Health. 1992, RAND Corporation: Santa Monica.

10. Lohr, K., et al., Use of Medical Care in the RAND Health Insurance Experiment, in Diagnosis- and Service-Specific Analyses in a Randomised Control Trial. 1986, RAND: Santa Monica. 
11. Manning, W., et al., Health Insurance and the Demand for Medical Care: Evidence from a Randomized Experiment. American Economic Review, 1987. 77(3): p. 251-277.

12. Taubman, S., et al., Medicaid Increases Emergency-Department Use: Evidence from Oregon's Health Insurance Experiment. Science, 2014. 343(6168): p. 263-268.

13. Allen, H., et al., The Oregon Health Insurance Experiment: When Limited Policy Resources Provide Research Opportunities. Journal of Health Politics, Policy and Law, 2013.

14. Finkelstein, A., et al., The Oregon Health Insurance Experiment: Evidence from the First Year The Quarterly Journal of Economics, 2012.

15. European Commission, The 2015 Ageing Report, in Economic and budgetary projections for the 28 EU Member States (2013-2060). 2015, European Commission: Brussels.

16. CSO, Population and Labour Force Projections in 2016-2046. 2013, CSO: Dublin.

17. National Consumer Agency, Doctor and Dentists Survey, in May 2010. 2010, National Consumer Agency: Dublin.

18. HSE, 2015 Medical Card and GP Visit Card Income Guidelines. 2015, HSE: Dublin.

19. HSE, Health Service Performance Report, in November Performance Report Supplementary Commentary. 2015, HSE: Dublin

20. Health Insurance Authority, February Newsletter 2016, Health Insurance Authority: Dublin.

21. Nolan, A., et al., The Over 50s in a Changing Ireland: Economic Circumstances, Health and Well-Being. 2014, The Irish Longitudinal Study on Ageing (TILDA): Dublin.

22. Whelan, B.J. and G.M. Savva, Design and Methodology of The Irish Longitudinal Study on Ageing. Journal of the American Geriatrics Society, 2013. 61: p. S265-S268.

23. Kearney, P., et al., Cohort Profile: The Irish Longitudinal Study on Ageing. International Journal of Epidemiology, 2011. 40(4): p. 877-884. 
24. Cronin, H., et al., Health and Aging: Development of The Irish Longitudinal Study on Ageing Health Assessment. Journal of the American Geriatrics Society, 2013. 61: p. S269-S278.

25. Hudson, E. and A. Nolan, Public Healthcare Eligibility and the Utilisation of GP Services by Older People in Ireland. Journal of the Economics of Ageing, 2015. 6: p. 24-43.

26. McNamara, A., C. Normand, and B. Whelan, Patterns and Determinants of Healthcare Utilisation in Ireland. 2013, TILDA: Dublin.

27. Ma, Y. and A. Nolan, Public Healthcare Entitlements and Healthcare Utilisation among the Older Population in Ireland. Health Economics, 2016. http://dx.doi.org/10.1002/ hec.3429.

28. Jiménez-Martín, S., J. Labeaga, and M. Martínez-Granado, An empirical analysis of the demand for physician services across the European Union. The European Journal of Health Economics, formerly: HEPAC, 2004. 5(2): p. 150-165.

29. Hoeck, S., et al., Healthcare utilisation among the Belgian elderly in relation to their socio-economic status. Health Policy, 2011. 99(2): p. 174-182.

30. Richardson, K., et al., Agreement between patient interview data on prescription medication use and pharmacy records in those aged older than 50 years varied by therapeutic group and reporting of indicated health conditions. Journal of Clinical Epidemiology, 2013. 66(11): p. 1308-1316.

31. Reijneveld, S. and K. Stronks, The validity of self-reported use of health care across socioeconomic strata: a comparison of survey and registration data. International Journal of Epidemiology, 2001. 30(6): p. 1407-1414.

32. Cleary, P. and A. Jette, The Validity of Self-Reported Physician Utilization Measures. Medical Care, 1984. 22(9): p. 796-803.

33. Fast, J. and D. Williamson, Poverty and medical treatment: when public policy compromises accessibility. Canadian Journal of Public Health, 1998. 89(2): p. 120-124. 
34. Stabile, M., Private Insurance Subsidies and Public Health Care Markets: Evidence from Canada. Canadian Journal of Economics, 2001. 34(4): p. 921-942.

35. Allin, S. and J. Hurley, Inequity in publicly-funded physician care: what is the role of private prescription drug coverage? Health Economics, 2009. 18(10): p. 1218-1232.

36. Nolan, A. and S. Smith, The effect of differential eligibility for free GP services on GP utilisation in Ireland. Social Science \& Medicine, 2012. 74(10): p. 1644-1651.

37. Rice, T. and K. Matsuoka, The Impact of Cost-Sharing on Appropriate Utilization and Health Status: A Review of the Literature on Seniors. Medical Care Research and Review, 2004. 61(4): p. 415-452.

38. Shigeoka, H., The Effect of Patient Cost Sharing on Utilization, Health, and Risk Protection. American Economic Review, 2014. 104(7): p. 2152-84.

39. Tamblyn, R., R. Laprise, and J. Hanley, Adverse events associated with prescription drug cost-sharing among poor and elderly persons. Journal of the American Medical Association, 2001. 285(4): p. 421-429.

40. Chandra, A., J. Gruber, and R. McKnight, Patient Cost-Sharing and Hospitalization Offsets in the Elderly. American Economic Review, 2010. 100(1): p. 193-213.

41. Chernew, M. and J. Newhouse, What does the RAND Health Insurance Experiment tell us about the impact of patient cost-sharing on health outcomes? American Journal of Managed Care, 2008. 14(7). 